\title{
The influence of prior knowledge in intentional versus incidental concept learning
}

\author{
WILLIAM D. WATTENMAKER \\ New Jersey City University, Jersey City, New Jersey
}

\begin{abstract}
Previous research has demonstrated that background knowledge has a clear influence on concept learning. This influence, however, has been observed with a narrow range of intentional learning tasks. In the present experiments, the role of background knowledge was examined as a function of a variety of incidental learning tasks as well as with intentional learning tasks. The influence of prior knowledge was investigated by comparing the encoding of conceptually related co-occurrences with the encoding of conceptually unrelated co-occurrences. A clear influence of prior knowledge was observed with incidental encoding, and conceptual relatedness was found to have at least as powerful an influence with incidental as with intentional learning tasks. The results indicate that many types of knowledgebased influences will not vary as a function of encoding strategy. The pervasiveness and strength of the influence of background knowledge on concept learning are discussed.
\end{abstract}

A major theme in categorization research has been the influence that background knowledge structures have on concept formation and categorization (e.g., Heit, 1994; Murphy \& Allopena, 1994; Murphy \& Medin, 1985; Waldmann, Holyoak, \& Fratianne, 1995; Ward, 1994; Wattenmaker, 1995; Wattenmaker, Dewey, Murphy, \& Medin, 1986; Wisniewski \& Medin, 1994). A second recent theme in categorization has been concerned with the types of concepts that are formed as a function of different encoding tasks, especially incidental and intentional tasks (see, e.g., Anderson \& Fincham, 1996; Brooks, 1978, 1987; Elio \& Anderson, 1984; Elio \& Lin, 1994; Kemler Nelson, 1984, 1988; Regehr \& Brooks, 1993; Ward \& Becker, 1992; Wattenmaker, 1991, 1993; Whittlesea, 1987). Research in these two areas has proceeded independently, however, and interactions between knowledge-based influences and encoding strategies have not been explored.

The research in this paper was designed to compare knowledge-based influences in intentional learning tasks with knowledge-based influences in incidental learning tasks. The terms knowledge structures and theories refer to schemata, mental models, informal theories, rules, causal relations, and general knowledge about the world (Murphy \& Medin, 1985). Intentional concept learning refers to cases in which the learner has the goal to learn about a category (or categories) during encoding. With intentional concept learning, people tend to actively test hy-

This research was supported in part by NIMH Grant MH45585. I thank Peter Dixon, Doug Nelson, and an anonymous reviewer for comments on this article. Correspondence concerning this article should be addressed to W. D. Wattenmaker, Department of Psychology, New Jersey City University, 2039 Kennedy Boulevard, Jersey City, NJ 07305 (e-mail: wwattenmaker@njcu.edu).

-Accepted by previous associate editor Peter Dixon potheses in order to uncover rules or generalizations that determine category membership (see, e.g., Brooks, 1978; Levine, 1975; Wattenmaker, 1991, 1993). With incidental concept learning, people process individual instances without intending to learn about a category, and indeed often there is no awareness of the category assignments of examples during encoding. The encoding task involves processing individual examples, but the goal of learning about a category or categories is not the focus of encoding. Consequently, systematic hypothesis testing and attempts to form rules that describe the categories do not occur with incidental encoding.

Background or prior knowledge has been found to have a powerful influence in concept learning and in sorting paradigms (e.g., Medin, Wattenmaker, \& Hampson, 1987; Murphy \& Allopena, 1994; Pazzani, 1991; Spalding \& Murphy, 1996; Ward, 1994; Wattenmaker, 1995; Wattenmaker et al., 1986; Wisniewski \& Medin, 1994). However, all of the experiments that have investigated influences of background knowledge have used intentional tasks. In learning paradigms, regardless of whether feedback was or was not presented during learning, examples have been presented as instances of a category (or categories) and the processing objective has been to learn about the category. Likewise, in sorting tasks, the goal has been to divide examples into categories. Thus, there is no evidence on whether an influence of background knowledge will be observed with incidental tasks or whether background knowledge has the same degree of influence with incidental as with intentional tasks.

Although there have been no investigations of the influence of prior knowledge with incidental encoding, in natural learning conditions people are often exposed to examples of categories under incidental conditions in which they are not strategic problem solvers. Consequently, it is especially important to investigate knowledgebased influences in incidental conditions. The experiments 
in this paper were designed to determine whether background knowledge structures have the same type and degree of influence in incidental as in intentional learning conditions.

\section{Relations Between Encoding Strategies and Knowledge Structures}

Research that has compared different encoding strategies has found that different strategies often produce clear differences in classification performance (e.g., Brooks, 1978; Kemler Nelson, 1984; Medin \& Smith, 1981; Nosofsky, Clark, \& Shin, 1989; Wattenmaker, 1991, 1993). Wattenmaker (1993), for example, compared incidental with intentional learning in knowledge-poor contexts, and differences in sensitivity to feature co-occurrences were observed as a function of the characteristics of incidental and intentional tasks.

There are several ways in which different learning strategies can produce differences in what is encoded and in classification behavior. The dimensions of the exemplars that are attended to, for instance, can vary with different encoding tasks, and the dimensions that are attended to are likely to influence the information that is retrieved and applied to the learning task. Different encoding strategies can also produce differences in the extent to which people actively engage in reasoning, elaborative, and inferential processes and in the extent to which there are deliberate attempts to search for and retrieve relevant information. These differences can also influence the knowledge that is activated and applied during learning. Thus, there is evidence that incidental and intentional tasks can produce clear differences in encoding and classification in knowledge-poor contexts, and if different encoding strategies activate different knowledge, these differences might be even greater in knowledgerich contexts.

If differences in the influence of background knowledge do occur with intentional and incidental encoding, what types of differences might be expected? There are a number of reasons to expect that the influence of background knowledge will be greater with intentional than with incidental tasks. Subjects in intentional conditions adopt a problem-solving mode in which they actively test hypotheses and seek generalizations. In the process of generating hypotheses, it seems likely that the subjects will draw on past knowledge and actively search memory for relevant information. This process of searching for related information and generating and testing alternative hypotheses would seem to be likely to activate a wide range of relevant knowledge that could be applied to the task. Thus, the active, problem-solving characteristics of intentional strategies might be more likely to activate and engage a wide base of background knowledge, and consequently the influence of relevant knowledge structures might be greater in intentional than in incidental conditions.

An alternative possibility is that the processes associated with activating, retrieving, and applying background knowledge will not be influenced by encoding strategy. In this perspective, the activation and application of relevant knowledge structures will occur spontaneously, and as long as subjects attend to the features of examples, relevant knowledge will be activated and applied to the task. Indeed, knowledge that is associated with attended information often seems to be retrieved spontaneously, and people have a basic tendency to connect new information to existing knowledge and to seek and develop interpretations and explanations in terms of prior knowledge. Consequently, similar types of knowledge might be activated and applied regardless of the processing objectives that operate during encoding. If this strategy-independent perspective is correct, the form and extent of knowledgebased influences will be the same with incidental and with intentional encoding tasks.

Clearly, in some cases relevant knowledge might be difficult to access, and in such cases the knowledge might not be applied to the task. However, the basic premises of the strategy-independent view are that (1) there is an enormous amount of background knowledge that is easily accessible and (2) this knowledge will be activated and applied without any effort or intention to retrieve and apply the information. Thus, a major prediction of the strategy-independent perspective is that if information is accessed and applied with one type of encoding task, it will be accessed and applied with other encoding tasks, and consequently the nature of knowledge-based influences will not vary as a function of strategy.

Whether the intentional-superiority perspective (i.e., the view that background knowledge will have a greater influence with intentional than with incidental encoding) or the strategy-independent perspective is more accurate might depend on the specifics of the encoding task and on the nature of the knowledge that must be applied. The terms knowledge structures and theories describe a great variety of types of knowledge, from isolated facts to coherent bodies of knowledge to abstract reasoning schemata. It is possible that some types of knowledge-based influences will be strategy independent, whereas other types will be strategy dependent. These experiments were designed to investigate whether the form and extent of knowledge-based influences are consistent across different encoding strategies and to evaluate whether the intentional-superiority or the strategy-independent perspective more accurately describes the relationship between knowledge and encoding strategy.

\section{EXPERIMENT 1}

In Experiment 1, the subjects were presented with descriptions that had conceptually related or conceptually unrelated feature co-occurrences. Conceptually related co-occurrences consisted of features that could be connected or perceived as related on the basis of background knowledge (e.g., has a heavy protective plate and moves slowly). Conceptually unrelated co-occurrences consisted of features that would be difficult to connect with back- 
ground knowledge (e.g., has a heavy protective plate and an adequate sense of smell). Descriptions with related and unrelated co-occurrences were presented in intentional or incidental learning conditions, and after the learning task a series of transfer tests was presented in order to determine the extent to which the co-occurrences had been encoded. If background knowledge that links the related co-occurrences is applied, there should be greater sensitivity to the conceptually related than to the conceptually unrelated co-occurrences on the transfer tests. Of greater interest, however, is the sensitivity to the conceptually related co-occurrences that will be observed in the incidental condition relative to the intentional condition. If background knowledge has as great an influence in incidental as in intentional encoding conditions, the increase in sensitivity to the related co-occurrences (relative to the unrelated co-occurrences) should be the same in incidental and intentional conditions.

The ability to encode co-occurrences has been hypothesized to be important for learning in many different areas of psychology including concept formation (e.g., Billman \& Knutson, 1996; Gluck \& Bower, 1988; Malt \& Smith, 1984; Murphy \& Wisniewski, 1989; Rosch, 1978). Although co-occurrences can be difficult to detect in some conditions (see, e.g., Murphy \& Wisniewski, 1989; Wattenmaker, 1991, 1993), many studies have found that people are fairly adept at detecting co-occurrences. Specifically, there is evidence that people are sensitive to unrelated co-occurrences in intentional conditions (e.g., Anderson \& Fincham, 1996; Estes, 1986; Medin, Altom, Edelson, \& Freko, 1982; Wattenmaker, $1991,1992,1993)$ as well as in incidental conditions (e.g., Wattenmaker, 1991, 1993; Wattenmaker, McQuaid, \& Schwertz, 1995). There is also evidence that people are sensitive to related co-occurrences in intentional conditions (e.g., Medin et al., 1987; Murphy \& Wisniewski, 1989; Wattenmaker, 1992). There is no evidence, however, on the sensitivity people demonstrate to related cooccurrences in incidental learning conditions, nor is there any research that has compared the encoding of related co-occurrences in incidental conditions with the encoding of related co-occurrences in intentional conditions.

\section{Learning Descriptions}

There were eight learning examples, and each example consisted of a list of five features (one feature from each of five dimensions). The co-occurrences were between features on two of the five dimensions, and the features on the other three dimensions were unrelated to the co-occurrences. Descriptions from three different domains were used to increase the generality of the results. The examples were descriptions of medical symptoms, descriptions of animals, or descriptions of people. Several different related and unrelated co-occurrences were used within each of these domains.

Table 1 shows the abstract structure that was used to construct the learning examples. This structure was used to construct learning examples that had co-occurrences on
Table 1

The Abstract Structure of the Examples for the D3-D5 Randomization in Experiments 1 and 2

\begin{tabular}{cccccc}
\hline & \multicolumn{5}{c}{ Dimension } \\
\cline { 2 - 6 } Exemplar & D1 & D2 & D3 & D4 & D5 \\
\hline 1 & 1 & 1 & 1 & 0 & 1 \\
2 & 0 & 0 & 1 & 1 & 1 \\
3 & 0 & 0 & 1 & 0 & 1 \\
4 & 1 & 1 & 1 & 1 & 1 \\
5 & 0 & 1 & 0 & 1 & 0 \\
6 & 1 & 0 & 0 & 1 & 0 \\
7 & 1 & 0 & 0 & 0 & 0 \\
8 & 0 & 1 & 0 & 0 & 0 \\
\hline
\end{tabular}

the third (D3) and fifth (D5) dimensions. The set of examples that was presented to a subject had either related or unrelated co-occurrences on the correlated dimensions. Appendix A, for example, illustrates a set of descriptions that was constructed from Table 1 and presented to subjects in the related conditions (i.e., conditions in which the co-occurring features were conceptually related). Appendix B illustrates a set of descriptions that were constructed from Table 1 and presented in the unrelated conditions (i.e., conditions in which the co-occurring features were not conceptually related). The descriptions in Appendix A and in Appendix B represent one of several different sets of descriptions that were used in the experiment.

For the materials in Appendix A, the co-occurring features were on the third and fifth dimensions, and as is shown in Appendix A, the co-occurring features were abdominal pain and indigestion in Exemplars 1-4 and fever and fatigue in Exemplars 5-8. Thus, a rule of the following form would correctly predict category membership of all the examples: If an example contains abdominal pain and indigestion or fever and fatigue, it is a member of the category. As is illustrated by the descriptions in Appendix B, the co-occurrences in the unrelated conditions had the same form as the co-occurrences in the related conditions but they were unrelated. In these descriptions, abdominal pain always co-occurred with gum disease, and fever always co-occurred with hair loss.

\section{The Encoding Tasks}

The subjects in the intentional conditions were informed that they were going to see descriptions that belonged to a common group and that they should carefully examine the descriptions and attempt to develop a rule that could be used to predict membership in this group. The instructions in the incidental conditions varied, depending on whether the descriptions were of medical symptoms, animals, or people. In all of the incidental conditions, however, the subjects were not told that the descriptions formed a group or category, nor were they told to attempt to search for rules or regularities in the descriptions. Instead, the subjects in the incidental conditions were asked to rate the descriptions.

For the descriptions of medical symptoms, the incidental task was to rate each description for how serious the 
ailment appeared to be; for the descriptions of people, the incidental task was to rate each description for how enjoyable it would be to interact with a person with these features; and for the descriptions of animals, the subjects rated each description for how much they would like to have the animal as a pet. The subjects in the incidental conditions were led to believe that the only purpose of the experiment was to rate the descriptions.

The rating tasks in the incidental conditions can be viewed as representing three similar but different incidental tasks in that they require rating different types of materials on different dimensions. In addition, each of the stimulus types is part of a different knowledge base, and it is possible that knowledge in different domains will vary in accessibility and structure (Wattenmaker, 1995). Thus, the data from the three stimulus types can be used to evaluate the generality of the results.

\section{Transfer Tests}

A series of transfer tests was presented after the learning phase to measure the extent to which the co-occurrences had been encoded. For each transfer test, two new descriptions were presented simultaneously, and the subjects were asked to select the description that was most consistent with the examples they had analyzed (i.e., intentional conditions) or rated (incidental conditions). The subjects in the intentional conditions anticipated a classification test of this type. The subjects in the incidental conditions, however, thought that their only task was to rate the descriptions; thus, they were unlikely to anticipate these tests.

The logic of the transfer tests was the following. Of the two descriptions that were presented for each of the tests, one of the descriptions preserved the co-occurrences that occurred in the learning examples, whereas the other description did not preserve the co-occurrences. Thus, if the co-occurrences were encoded and influenced decisions, the subjects should be more likely to select the transfer item that preserved the co-occurrences than to select the item that broke the co-occurrences. Twenty transfer tests of this form were presented.

The abstract notation of the 20 transfer tests that were presented in the related and unrelated conditions is shown in Table 2. The first items listed in Table 2 (i.e., the items in the A column) always preserved the cooccurrences (they had the pattern 11 or 00 on the third and fifth dimensions). The second items listed in Table 2 (i.e., the items in the B column) always broke the cooccurrences (these items had 10 or 01 on the third and fifth dimensions). Thus, if the co-occurrences were encoded and influenced decisions, there should be a greater proportion of $A$ items than of $B$ items selected.

\section{The Control Condition}

A control condition was also tested. The subjects in the control condition were given the same transfer tests and made the same transfer decisions as the subjects in
Table 2

Transfer Tests for the D3-D5 Randomization in Experiments 1 and 2

\begin{tabular}{ccc}
\multicolumn{3}{c}{ in Experiments 1 and 2 } \\
\cline { 2 - 3 } Test & A & B \\
\hline 1 & 01111 & 01011 \\
2 & 11000 & 11100 \\
3 & 10111 & 10011 \\
4 & 00010 & 00110 \\
5 & 10101 & 10100 \\
6 & 00000 & 00001 \\
7 & 01101 & 01100 \\
8 & 11010 & 11011 \\
9 & 01111 & 10011 \\
10 & 11000 & 00110 \\
11 & 10111 & 10100 \\
12 & 00010 & 11011 \\
13 & 10101 & 01100 \\
14 & 00000 & 01011 \\
15 & 01101 & 00001 \\
16 & 00010 & 11100 \\
17 & $--1-1$ & $--1-0$ \\
18 & $--1-1$ & $-0-1$ \\
19 & $-0-0$ & $-1-0$ \\
20 & $-0-0$ & $-0-1$ \\
\hline
\end{tabular}

the intentional and incidental conditions, but they never saw the learning descriptions and did not go through a learning phase. The purpose of the control condition was to provide a baseline of the influence of the related cooccurrences on transfer decisions. Specifically, if the conceptual relatedness of the co-occurrences made the related co-occurrences highly salient in the transfer items, it is conceivable that test descriptions with related cooccurrences might be preferred to test descriptions with unrelated co-occurrences even if there was no exposure to the learning descriptions. If this occurred, the subjects in the control condition should be more likely to select the test alternatives with related co-occurrences than the alternatives without related co-occurrences. Thus, this control condition will be useful for interpreting the results of the intentional-related and incidental-related conditions.

\section{Summary}

If relevant knowledge structures are activated and applied in incidental learning tasks, greater sensitivity to the co-occurrences should be observed in the incidentalrelated condition than in the incidental-unrelated condition. In addition, if the influence of background knowledge is as great in incidental as in intentional conditions, the increase in sensitivity to the related co-occurrences should be as great in the incidental as in the intentional conditions.

\section{Method}

Subjects. The subjects were 229 undergraduates, who participated in the experiment to fulfill course requirements. The intentional- 
related, intentional-unrelated, incidental-related, and incidentalunrelated conditions each had 24 subjects. The control-related and control-unrelated conditions each had 48 subjects. Thirty-seven subjects participated in a rating task.

Stimuli. Different related co-occurrences were used in different randomizations. The 12 related co-occurrences that were used were abdominal pain and indigestion, fever and fatigue, weight gain and high blood pressure, shortness of breath and chest pain, has a heavy protective plate and moves slowly, has sharp teeth and is a carnivore, sleeps in the day and lives in a well-hidden home, has wings and flies, intelligent and inquisitive, friendly and warm, arrogant and selfconfident, and lively and talkative. The 12 unrelated co-occurrences were abdominal pain and gum disease, fever and hair loss, weight gain and low white cell count, shortness of breath and a twisted ankle, has a heavy protective plate and has an adequate sense of smell, sharp teeth and oval eyes, sleeps in the day and has an adequate sense of sight, has wings and has oval eyes, intelligent and unpredictable, friendly and disorganized, arrogant and relaxed, and lively and crafty.

A separate group of 37 subjects evaluated the relatedness of the co-occurrences. They were presented with pairs of features and were asked to indicate how likely it was that each pair of features would occur together. Each pair of features was rated on a scale from 1 (rarely occur together) to 7 (often occur together). The average rating for the 12 related co-occurrences was 5.70 , and the average rating for the 12 unrelated co-occurrences was 2.84 .

The learning examples were constructed for a particular stimulus type (e.g., medical symptoms) by first selecting one of the cooccurrences for that stimulus type. For example, for the medical descriptions in the related condition, abdominal pain and indigestion might be selected. Then, in terms of Table 1 , one of the features (e.g., abdominal pain) was assigned the value 1 on $\mathrm{D} 3$, and the other feature (e.g., indigestion) was assigned the value 1 on D5. Then, another pair of related features from this domain was selected (e.g., fever and fatigue), and one of these features (e.g., fever) was assigned the value 0 on D3, and the other feature (e.g., fatigue) was assigned the value 0 on D5. Features that were unrelated to the cooccurrences were assigned to the irrelevant dimensions (D1, D2, and D4, in the case of Table 1).

Across subjects the co-occurrences occurred on four different pairs of dimensions. The notation in Table 1 illustrates the case in which the co-occurring features were on the third and fifth dimensions (i.e., the third and fifth features that were listed). For other subjects, however, the co-occurrences were between the first (D1) and fourth (D4) dimensions, the second (D2) and third (D3) dimensions, or the fourth (D4) and fifth (D5) dimensions. The category structure for the randomizations in which the co-occurrences were on D1-D4, D2-D3, and D4-D5 was identical to that in Table 1, except that the co-occurrences were between different dimensions. The structure in Table 1 was designed so that all individual features occurred equally often. All pairs of features, other than the cooccurrences, also occurred equally often.

Within each condition (e.g., the incidental-related condition), the 12 co-occurrences occurred equally often. Each of the 12 cooccurrences also occurred equally often on the four correlated dimensions (i.e., D1-D4, D2-D3, D3-D5, and D4-D5). These counterbalancing procedures produced 24 different sets of learning descriptions in the related conditions and 24 different sets of learning examples in the unrelated conditions.

As was noted, the abstract notation of the transfer tests is shown in Table 2. An example of a transfer test that was presented for the learning descriptions in Appendix B was low plasma level, brittle bones, abdominal pain, twisted ankle, and gum disease versus low plasma level, reduced visual acuity, fever, twisted ankle, and gum disease. In terms of the learning examples in Appendix B, the first of these descriptions preserves the co-occurrence between abdominal pain and gum disease, whereas the second description breaks the co-occurrence, in that the features fever and gum disease never occurred together in the learning examples. An example of a transfer test for the materials with related co-occurrences in Appendix A was reduced visual acuity, twisted ankle, fever, gum disease, and fatigue versus brittle bones, low sodium level, fever, gum disease, and indigestion. In terms of the learning examples in Appendix A, the first description preserves the co-occurrence between fever and fatigue, whereas the second description has the features fever and indigestion, which never co-occurred in the learning descriptions.

Transfer Tests 1-16 (Table 2) included features from all five dimensions, whereas the partial-information tests (Tests 17-20) only included features from the correlated dimensions (e.g., for the materials in Appendix B, Test 17 was represented as: -----, -----, abdominal pain, -----, gum disease versus -----, -----, abdominal pain, ----, hair loss). The partial-information tests were always presented after the full-information tests, to avoid drawing attention to the cooccurrences.

Comparison of test items. Tests $1-8$ in Table 2 were designed to provide a particularly sensitive measure of the influence of cooccurrences. Specifically, Tests 1 and 2,3 and 4, 5 and 6 , and 7 and 8 were matched to reveal the influence of the co-occurrences. For example, the two descriptions in Test 1 (01111 vs. 01011$)$ only differ on the value of the third dimension. If the $\mathrm{A}$ item is selected in Test 1 , this could reflect either an influence of the co-occurring features on D3 and D5 or a preference for the feature represented by the value 1 on D3 over the feature represented by the value 0 on D3. The alternatives in Test $2(11000$ vs. 11100$)$ also only differ on the value of the third dimension. However, in this case, if the feature represented by the value 1 rather than the co-occurrence was the basis for selecting the A item in Test 1 , the B item should be selected in Test 2. Alternatively, if the co-occurrences were guiding decisions, the A alternative should be selected on Test 2 and on Test 1 . In general, if there is a clear preference for the $A$ item on Test 1 and also on Test 2, it would not be possible to explain the results in terms of the salience of individual features, and the results would provide very strong support for the influence of co-occurrences. Tests 3-4, $5-6$, and 7-8 set up these same types of contrasts.

Procedure. The learning descriptions were presented individually in five blocks; a block consisted of presentation of all eight learning descriptions. Each presentation of a description was for $20 \mathrm{sec}$. The descriptions were presented in a booklet that had one description per page and that consisted of 40 pages (five presentations of the eight descriptions). Within each block, the eight descriptions were presented in a random order. Across blocks, the presentation of the same description was always separated by at least one other description.

In the intentional conditions, after the descriptions had been studied for $20 \mathrm{sec}$, the subjects were instructed to immediately turn to the next description. As was noted, the instructions in the incidental conditions varied for the medical, animal, and people materials. For all materials, however, the subjects read the description for $20 \mathrm{sec}$, and they were then instructed to immediately make their rating and turn to the next description. The subjects in the incidental conditions provided ratings for all 40 presentations of the learning descriptions. The rating scale varied from 1 (e.g., would not like at all) to 7 (e.g., would like very much).

After the learning descriptions had been presented, the subjects were given a booklet with the 20 transfer tests. Each page had 1 transfer test. The two alternative descriptions for a transfer test (e.g., as represented by Test 1 in Table 2) were presented together on a page, and the subjects were instructed to select the description that was most consistent with the descriptions they had analyzed (intentional conditions) or rated (incidental conditions). The location of the test description that preserved the co-occurrences was randomly determined. There was no time limit for the transfer decisions, and no feedback was provided. The transfer tests were presented in a ran- 
dom order (except that the partial-information tests were always presented after the full-information tests). The subjects were not allowed to look at previous or future items in either the learning or the test booklets. After the transfer phase, the subjects were asked to indicate (through written responses) how they made their classification decisions and whether they noticed any rules or regularities.

The subjects in the control conditions only performed the transfer task. They were informed that they were going to see pairs of descriptions, that one of the descriptions belonged to a group or category, and that their task was to select the correct description. They were told that since they did not know what the group was, they should carefully examine the descriptions and then make an educated guess about which description to select. There was no time limit on decisions, and no feedback was presented.

\section{Results}

The proportion of tests in which the alternative with co-occurring features was selected for Transfer Tests 1-16 (i.e., the full-information tests) is shown in Figure 1 . These results indicate that there was greater sensitivity to the related than to the unrelated co-occurrences in both the intentional and the incidental conditions, and that conceptual relatedness was as beneficial in the incidental as in the intentional conditions. Planned comparisons revealed that the descriptions that preserved the cooccurrences were selected significantly more often in the incidental-related condition than in the incidentalunrelated condition $[.81$ vs. $.56 ; t(46)=4.68, p<.001$; $S E=.57$ in the incidental-related condition, and $S E=$ .66 in the incidental-unrelated condition]. The difference between the intentional-related and the intentionalunrelated conditions was also significant [.73 vs. .55; $t(46)=3.57, p<.001 ; S E=.59$ in the intentional-related condition, and $S E=.58$ in the intentional-unrelated condition]. The subjects in the incidental-related condition showed a slight tendency to be more sensitive to the related co-occurrences than did the subjects in the intentional- related condition, but this difference was not significant $[t(46)=1.52, p=.14]$. The interaction between encoding condition (intentional vs. incidental) and co-occurrence type (related vs. unrelated) also was not significant ( $p=.35$ ). The lack of an interaction reflects the observation that the influence of knowledge was as great in the incidental as in the intentional conditions.

The results of each of the individual conditions were compared with the relevant control condition to determine whether there was a significant influence of the cooccurrences above what would be expected if there was no sensitivity to or encoding of the co-occurrences during the learning phase. These comparisons revealed that the subjects in the incidental-related condition $[t(70)=$ $8.83, p<.001]$ and in the intentional-related condition $[t(70)=6.59, p<.001]$ demonstrated greater sensitivity to the co-occurrences than did the subjects in the controlrelated condition. The difference between the incidentalunrelated condition and the control-unrelated condition was not significant $[t(70)=1.25, p=.22]$. The difference between the intentional-unrelated and control-unrelated conditions also was not significant $[t(70)=1.12, p=.27]$.

The results of the individual transfer tests are shown in Appendix C. The number of the transfer test in Appendix $\mathrm{C}$ corresponds to the number of the transfer test

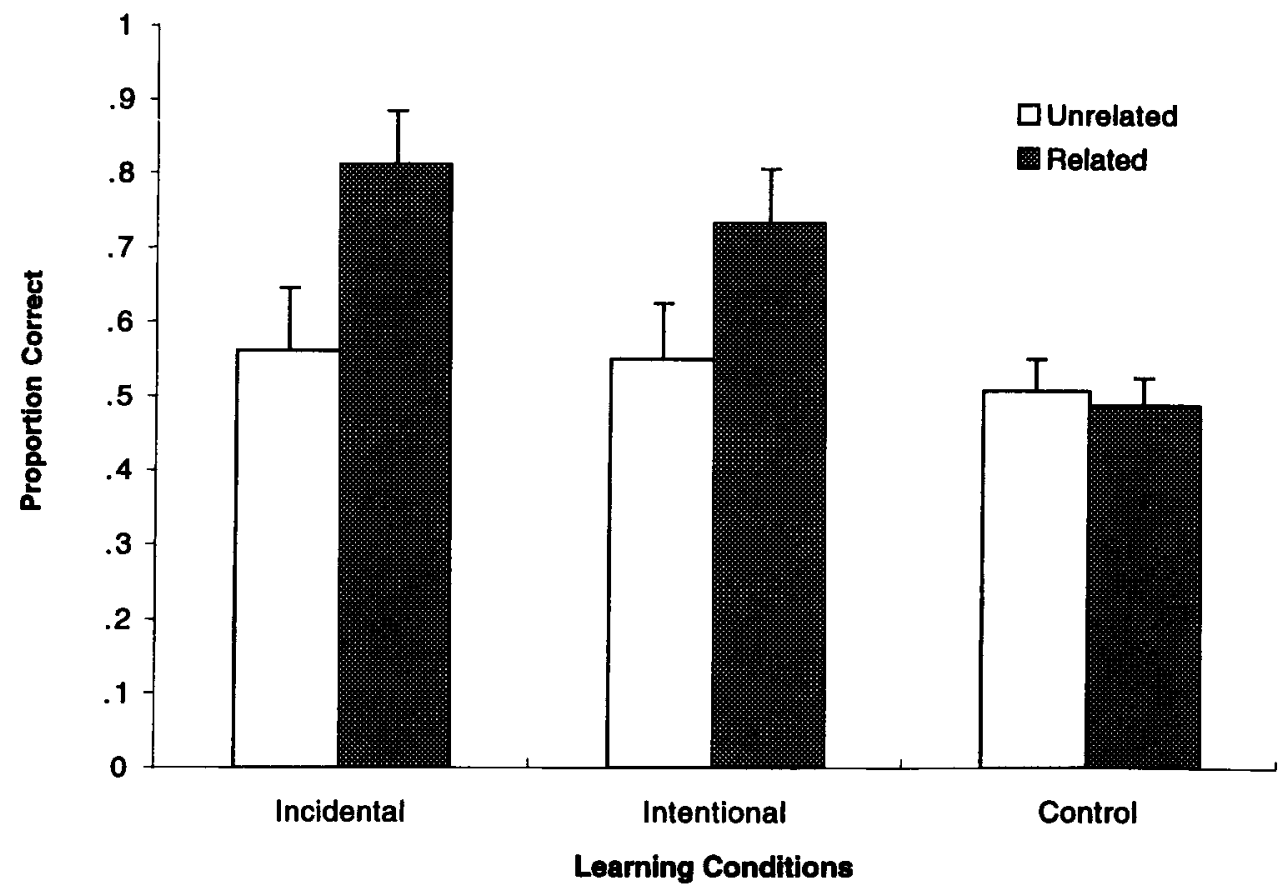

Figure 1. Proportions of responses in which transfer descriptions with co-occurring features were selected, as a function of encoding condition and type of co-occurrence in Experiment 1. 
in Table 2 (e.g., in Appendix C, the proportions next to Test 1 represent the proportion of the time that the description that represented the notation 01111 was selected over the description that represented the notation 01011). The proportions in Appendix $\mathrm{C}$ represent the combined results of all the randomizations. The overall results of Tests 1-16 were discussed above. The results of the partialinformation tests (17-20) should be interpreted with some caution, given that there was a small number of tests relative to the full-information tests. More important, the partial-information tests can be difficult to interpret in the related conditions because these tests included only the two related features, whereas the full-information tests consisted of a list of features on all the dimensions. Related co-occurrences should be much more salient when they are listed adjacently and when they are the only two features provided than when they are embedded in a list of domain-relevant features. Nonetheless, if the above analyses are repeated with the partial- and fullinformation tests combined, almost all of the results discussed above remain the same. The one slight exception is that rather than being suggestive, the difference between the incidental-unrelated condition and the controlunrelated condition was close to significance $[t(70)=1.96$, $p=.055]$. Given that only knowledge of co-occurrence frequencies could be used to guide decisions on the partialinformation tests in the unrelated conditions, these results indicate that the subjects in the incidental-unrelated condition encoded the co-occurrences during learning. (Incidentally, although the subjects in the control-related condition showed a tendency to select the related cooccurrences on the partial-information tests, as was discussed above the key result was that on the fullinformation tests, the subjects in the control-related condition were at chance (.49), whereas the subjects in the incidental-related (.81) and intentional-related (.73) conditions were way above chance.)

Results of the different stimulus types. Analysis of the data from the medical, animal, and people materials revealed that for all three types of stimuli and tasks, the subjects in the incidental-related condition were as sensitive to the co-occurrences as the subjects in the intentional-related condition. In the incidental-related condition, the test descriptions with related co-occurrences were selected $89 \%, 83 \%$, and $74 \%$ of the time for the medical, animal, and people descriptions, respectively. In the intentional-related condition, these results were $68 \%, 80 \%$, and $76 \%$; in the incidental-unrelated condition, they were $56 \%, 64 \%$, and $54 \%$; and in the intentionalunrelated condition, they were $49 \%, 56 \%$, and $61 \%$.

Results of the individual transfer tests. As was noted for the individual transfer tests in Table 2, Tests 1 and 2, 3 and 4,5 and 6 , and 7 and 8 were designed to be especially sensitive to the use of co-occurrences. These tests were matched so that if the description with co-occurring features was selected on both of the matched tests, the results would provide very strong support for the use of feature co-occurrences. Specifically, if the subjects were using single features and there was no influence of the co-occurrences, the combined results of the matched tests (i.e., Tests 1 and 2, 3 and 4,5 and 6, and 7 and 8) should not be above .50. An examination of the results in Appendix $\mathrm{C}$ shows that for the four pairs of matched tests, there was a very clear influence of the co-occurrences in the incidental-related condition, as the combined results of all of the matched tests were well above .50. (Across the four matched tests, there was an average difference of $31 \%$ in the direction that was consistent with the use of the co-occurrences.) This pattern of results was also observed in the intentional-related condition (an average difference of $21 \%$ in the direction consistent with the co-occurrences), but relatively little influence of the cooccurrences was observed in the incidental-unrelated, the intentional-unrelated, the control-related, and the control-unrelated conditions (average differences of $5 \%$, $4 \%, 2 \%$, and $2 \%$, respectively).

Salience of the related co-occurrences. The rating data indicated that the related co-occurrences differed considerably in salience. Potentially, the incidental tasks might have produced sensitivity to the highly salient related co-occurrences but not to the low-salience related co-occurrences. This possibility was evaluated by comparing the results of the classification tests that included the four most salient (i.e., highest rated) co-occurrences with the results of the tests that included the four least salient (i.e., lowest rated) co-occurrences. (The average rating for the four highest rated co-occurrences was 6.21 , and the average rating for the four lowest rated cooccurrences was 5.07.) This analysis indicated that the subjects in the incidental-related condition were as sensitive to the lowest rated related co-occurrences as to the highest rated related co-occurrences. The descriptions with the four highest rated co-occurrences were selected $80 \%$ of the time, and the descriptions with the four lowest rated co-occurrences were also selected $80 \%$ of the time. In addition, sensitivity to the low-salience cooccurrences was as great in the incidental-related condition as in the intentional-related condition; the lowsalience co-occurrences were again selected $80 \%$ of the time in both the incidental-related condition and the intentional-related condition. Thus, these results indicate that the influence of prior knowledge occurs in incidental tasks even if the co-occurrences are not especially salient. Indeed, comparison of the two highest and the two lowest rated related co-occurrences (average ratings of $6.36 \mathrm{vs.} 4.56$ ) indicated that the subjects in the incidental-related condition were approximately equally likely to select test alternatives with the two least salient co-occurrences as to select test alternatives with the two most salient co-occurrences (.73 vs. .75).

\section{Discussion}

Greater sensitivity to related than to unrelated cooccurrences was observed in the incidental and the in- 
tentional conditions, and conceptual relatedness was as beneficial in the incidental as in the intentional conditions. These results held for three different types of stimulus materials and for low-salience as well as for highsalience related co-occurrences. Thus, although it seemed probable that the active, problem-solving mode adopted with intentional strategies would be more likely to lead to the retrieval and application of relevant knowledge structures, the influence of background knowledge was as great in incidental as in intentional conditions. These results are consistent with the strategy-independent perspective outlined in the introduction of the paper: Relevant background knowledge appeared to be spontaneously applied, and the nature and extent of the influence of knowledge did not vary as a function of encoding strategy.

Although the subjects in the incidental-related condition were simply rating the descriptions and not attempting to search for rules, the test descriptions that preserved the co-occurrences were selected at a very high rate ( $81 \%$ of the time). For the related co-occurrences to have become salient during encoding, knowledge must have been retrieved that provided conceptual links between the co-occurring features. These conceptual linkages can take many forms, such as retrieving causal connections that explain why two features might be related, retrieving stored knowledge about co-occurrence frequencies in the environment, retrieving isolated facts that two features co-occur without an understanding of why (e.g., knowing that weight gain and blood pressure are linked without having a causal explanation of why they are linked), retrieving an extensive body of knowledge that links the features to each other and to related information (e.g., fever and fatigue could retrieve a category such as infection, which could be used to link the two features and to connect the features with related knowledge about infections), and generating or inferring (rather than retrieving) reasons why two features might be connected. There are important differences between these forms of conceptual relatedness, but the results of Experiment 1 raise the possibility that the background knowledge that produces many of these conceptual, causal, and inferential links will be spontaneously accessed and applied with a wide range of encoding strategies.

In addition to providing new evidence about the encoding of related co-occurrences with incidental processing, the results also solidify a number of other results concerning the encoding of co-occurrences. Specifically, the results support previous findings that co-occurrences that are conceptually related will be encoded more readily than unrelated co-occurrences in intentional conditions (Heit, 1994; Medin et al., 1987; Murphy \& Wisniewski, 1989). The results also support the finding that incidental encoding can capture unrelated co-occurrences (Wattenmaker, 1991, 1993; Wattenmaker et al., 1995).

\section{EXPERIMENT 2A}

Experiment 2 was designed to examine the generality of the results of Experiment 1 by using incidental tasks that were very different from the incidental tasks that were used in Experiment 1 . There are a variety of incidental tasks, and different tasks can vary in important ways. A potentially important aspect of an incidental task might be the degree to which the overall task engages general knowledge structures. In the case of learning co-occurrence relations, for example, some form of prior knowledge must provide connections between cooccurring features to increase their salience. Thus, incidental tasks that result in the activation of extensive background knowledge will probably increase the likelihood that information that links co-occurring features will be retrieved.

In this regard, it is likely that various types of general knowledge structures were activated in Experiment 1 as a by-product of the rating tasks. A task such as rating the seriousness of a set of medical symptoms, for example, might have resulted in a search for a disease that would fit the symptoms, and the ratings might have been based on the disease that was retrieved. Thus, the related cooccurrences might have become salient as a by-product of searching existing knowledge of diseases.

In Experiment 2, we attempted to use incidental tasks that minimized the likelihood that the subjects would retrieve prior knowledge that might be relevant to the cooccurrences. This was accomplished by using incidental tasks that were data driven or self-contained in the sense that the tasks required that subjects attend to the learning exemplars, but the need to retrieve prior knowledge was minimized.

Two different incidental tasks were used. In what will be referred to as the recognition condition, a series of descriptions was presented in the learning phase. The descriptions were presented individually, and for each description that was presented the only task was to decide whether the description had or had not been presented previously in the series. The subjects decided whether each description was old (i.e., had appeared previously in the series) or new (i.e., had not appeared previously in the series) and indicated their confidence on a scale from 1 (not very confident) to 7 (very confident). For the first description that was presented in the series, the correct response was clearly new, but for every description that was presented after the first one, the subjects had to decide whether the description had or had not occurred earlier in the sequence (e.g., for the tenth description that was presented, the question was whether this description was a repeat of any of the previous nine descriptions). The subjects were not told that the descriptions formed a group or category; their only task was to try to be accurate in their recognition judgments. This encoding task focuses attention on the learning examples, but it minimizes the involvement of prior knowledge in the sense that the subjects only have to rely on their recall of information that was presented in the experimental context to perform the task.

The second incidental task will be referred to as the $o b$ servation condition. In this condition, the subjects again were not informed that the descriptions constituted a 
group or category, nor were they instructed to search for rules or regularities. Instead, they were simply told to "look over and examine" the descriptions as they were presented. The subjects in the observation condition did not make any ratings; they simply observed the descriptions. Again, this condition only requires that the subjects attend to the descriptions, and it does not require that the subjects actively retrieve prior knowledge to perform the task.

The intentional conditions in this experiment were identical to the intentional conditions of Experiment 1. With the exception of the changes in the incidental tasks noted above, the procedures and materials in this experiment were identical to those used in Experiment 1.

The incidental tasks in this experiment are clearly very different from the incidental tasks that were used in Experiment 1. Thus, this experiment should provide a good test of the generality of the results of Experiment 1 . If knowledge that makes related co-occurrences salient is activated and applied regardless of the encoding strategy, greater sensitivity to related than to unrelated cooccurrences should be observed in the recognition and observation conditions.

\section{Method}

Subjects. The subjects were 144 undergraduates, who participated in the experiment for course credit. The subjects were randomly assigned to the recognition-related, recognition-unrelated, observation-related, observation-unrelated, intentional-related, or intentional-unrelated conditions.

Stimuli and Procedure. The category structures, stimulus materials, related and unrelated co-occurrences, and transfer tests were identical to those in Experiment 1. With the exception of the dif- ferences in the incidental tasks, all aspects of the learning and transfer procedures were also identical to those in Experiment 1.

\section{Results}

The combined results of Transfer Tests $1-16$ are shown in Figure 2. An examination of Figure 2 reveals that even with these very different incidental tasks, there was greater sensitivity to the related than to the unrelated co-occurrences in both of the incidental conditions. The proportion of the time that the alternatives with cooccurring features were selected was greater in the recognition-related than in the recognition-unrelated condition $[.75$ vs. $.64 ; t(46)=2.11, p<.05 ; S E=.57$ in the recognition-related condition, and $S E=.63$ in the recognition-unrelated condition]. The difference between the observation-related and the observation-unrelated conditions was also significant $[.72$ vs. $.57 ; t(46)=2.62$, $p=.01 ; S E=.66$ in the observation-related condition, and $S E=.65$ in the observation-unrelated condition]. Surprisingly, greater sensitivity to the related than to the unrelated co-occurrences was not observed in the intentional conditions $[.62$ vs. $.64 ; t(46)=0.47, p=.64 ; S E=$ .73 in the intentional-related condition, and $S E=.64$ in the intentional-unrelated condition]. Comparison of the recognition and the intentional conditions indicated that the interaction between encoding condition (recognition vs. intentional) and co-occurrence type (related vs. unrelated) was not significant $(p=.08)$, but the interaction between the observation and intentional conditions and the relatedness of the co-occurrences was significant $\left[F(1,92)=4.62, M S_{\mathrm{e}}=10.73, p<.05\right]$.

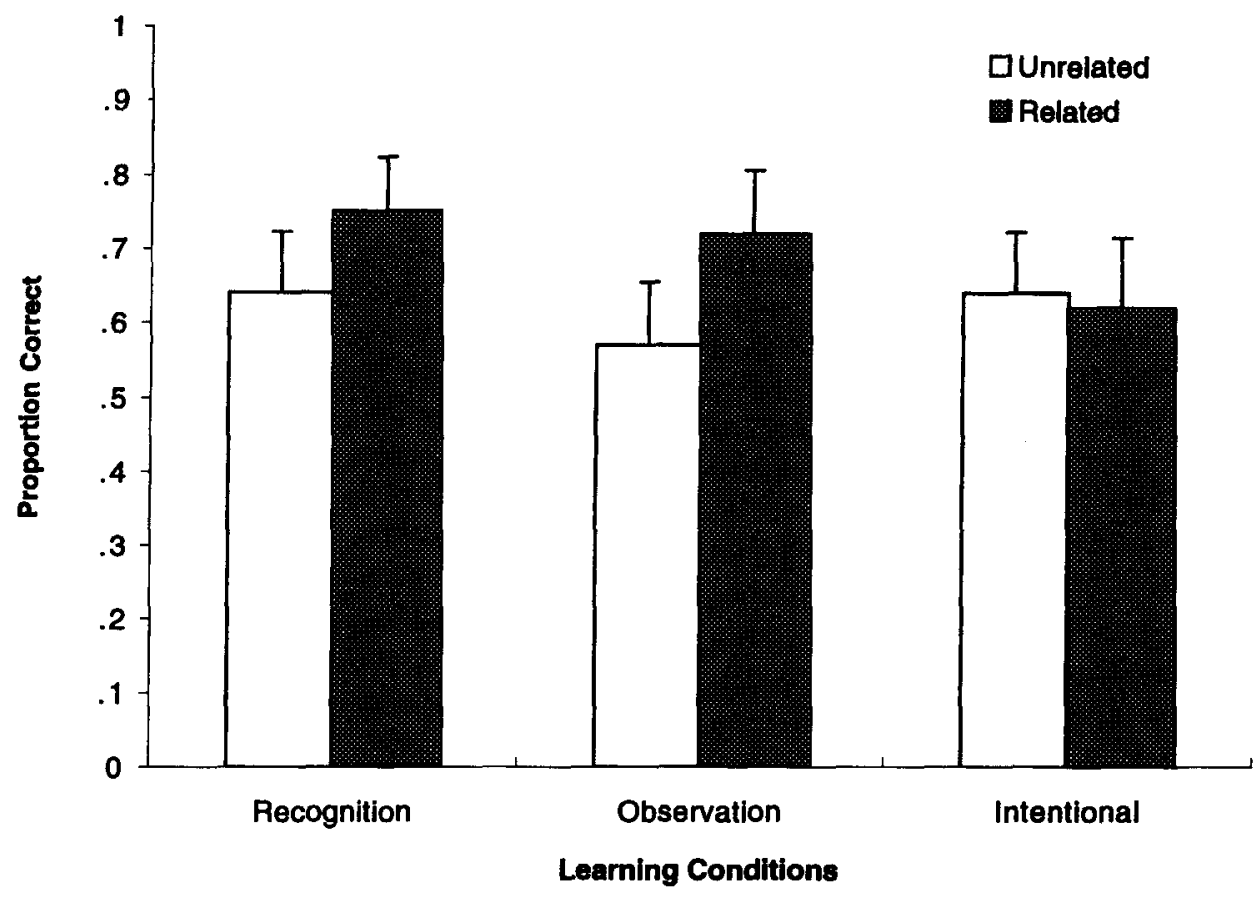

Figure 2. Proportions of responses in which transfer descriptions with co-occurring features were selected, as a function of encoding condition and type of co-occurrence in Experiment 2. 
Comparison of the related conditions revealed that the alternative that preserved the co-occurrences was selected significantly more often in the recognition-related condition than in the intentional-related condition [.75 vs. $.62 ; t(46)=2.35, p<.05]$. The difference between the observation-related and the intentional-related conditions approached significance $[.72$ vs. . $62 ; t(46)=1.74, p=$ $.09]$. There was no significant difference between any of the unrelated conditions.

The results of each of the conditions were compared with the control conditions to determine whether there was a significant influence of the co-occurrences in the individual conditions. The transfer tests in this experiment were identical to those in Experiment 1, and the control data collected in Experiment 1 were based on large numbers of subjects. Thus, the results of the control conditions reported in Experiment 1 were also used in this experiment. The comparisons between each of the conditions and the relevant control condition revealed that all of the conditions showed a significant influence of the cooccurrences. The alternatives that preserved the cooccurrences were selected significantly more often in the intentional-related than in the control-related condition $[t(70)=2.95, p<.01]$, in the recognition-related than in the control-related condition $[t(70)=7.11, p<.001]$, and in the observation-related than in the control-related condition $[t(70)=5.88, p<.001]$. In relation to the control- unrelated condition, the test alternatives that preserved the co-occurring features were selected significantly more often in the intentional-unrelated condition $[t(70)=3.35, p<.01]$ and in the recognition-unrelated condition $[t(70)=3.23, p<.01]$, but not in the observation-unrelated condition $[t(70)=1.57, p=.12]$.

The results of the individual transfer tests are shown in Appendix C. If the analyses of the full-information tests described above are expanded to include the partialinformation tests, then as in Experiment 1 almost all of the results are unchanged. The only exception is that rather than approaching significance, the difference between the observation-unrelated condition and the controlunrelated condition is now significant $[t(70)=1.98, p=$ $.05]$. This suggests that as in the recognition-unrelated and intentional-unrelated conditions, there was some sensitivity to the unrelated co-occurrences in the observationunrelated condition.

Results of the different stimulus types. The data from the medical, animal, and people materials revealed that the overall pattern of results held for each of the domains. For all three types of stimuli, the subjects in the incidental conditions showed equal or greater sensitivity to the related co-occurrences than did the subjects in the intentional condition. In the recognition-related condition, the proportion of the time that the alternative that preserved the co-occurrences was selected was $.81, .78$, and .76, for the medical, animal, and people descriptions, respectively. These proportions were $.72, .79$, and .74 in the observation-related condition, $.70, .65$, and .57 in the intentional-related condition, $.60, .69$, and .66 in the recognition-unrelated condition, $.62, .60$, and .53 in the observation-unrelated condition, and $.62, .69$, and .62 in the intentional-unrelated condition.

Results of the individual transfer tests. An examination of the matched Tests $1-2,3-4,5-6$, and 7-8 in Appendix $C$ reveals that there was an influence of cooccurring features in all of the conditions for both the related and the unrelated co-occurrences. Consistent with the overall results, the clearest influence of cooccurrences was observed in the recognition-related and observation-related conditions. For the four matched tests, there was an average difference of $.15, .29, .28, .16$, .11 , and .12 in the direction that was consistent with the use of co-occurrences in the intentional-related, observation-related, recognition-related, intentionalunrelated, observation-unrelated, and recognitionunrelated conditions, respectively.

Salience of the related co-occurrences. Examination of the four highest rated related co-occurrences and the four lowest rated related co-occurrences indicated that the subjects in the incidental conditions showed approximately equal sensitivity to the low- and the high-salience related co-occurrences. In the recognition-related, observation-related, and intentional-related conditions, the test alternatives that had the four least salient cooccurrences were selected $76 \%, 76 \%$, and $69 \%$ of the time, respectively, and the test alternatives that had the four most salient co-occurrences were selected $83 \%$, $70 \%$, and $59 \%$ of the time. (If the two least salient related co-occurrences are compared with the two most salient related co-occurrences, then again, the subjects in the incidental conditions were approximately equally sensitive to the two least salient and the two most salient cooccurrences [.74 vs. .76].)

\section{Discussion}

Even though very different incidental tasks were used in this experiment, the same pattern of results that occurred in the incidental conditions of Experiment 1 was observed: A greater influence of conceptually related than of conceptually unrelated co-occurrences was observed in both of the incidental conditions, and the influence of conceptual relatedness was as great or greater in the incidental than in the intentional conditions. The finding that a clear influence of knowledge was found with incidental tasks that were designed to minimize the influence of prior knowledge supports the conclusion that relevant knowledge structures will often be spontaneously applied during concept learning, regardless of the specifics of the encoding strategy.

Clear sensitivity to unrelated as well as to related cooccurrences was observed. These results indicate that co-occurrences do not have to be conceptually related to be encoded and are consistent with prior research that 
has found an influence of unrelated co-occurrences in intentional tasks (e.g., Medin et al., 1982) as well as in incidental tasks (e.g., Wattenmaker, 1991, 1993; Wattenmaker et al., 1995). People are often exposed to and need to encode co-occurrences before the reasons for the co-occurrences are known; thus, it clearly is important to have learning mechanisms that can encode unrelated co-occurrences.

Although there was clear sensitivity to the related cooccurrences in the intentional-related condition, no difference was found between the intentional-related and the intentional-unrelated conditions. This result was due, in part, to the pronounced sensitivity to unrelated cooccurrences that occurred in the intentional-unrelated condition. Further analysis of the intentional-unrelated condition revealed that 7 subjects explicitly reported forming and using rules that involved the co-occurrences and that these subjects selected the option that preserved the co-occurrences $83 \%$ of the time. The remaining subjects in the intentional-unrelated condition did not report detecting the co-occurrences, and these subjects selected the option with co-occurrences only $56 \%$ of the time. As these results illustrate, an advantage of intentional strategies is that if accurate rules are developed, they can lead to excellent transfer. Of course, if inaccurate rules or rules that have little diagnosticity are developed, transfer will be poor. In any event, the results of Experiment 1 and the results of previous research (e.g., Heit, 1994; Medin et al., 1987; Murphy \& Wisniewski, 1989) clearly indicate that greater sensitivity to related than to unrelated co-occurrences will usually be observed in intentional conditions. Medin et al. (1987, Experiment 5), for example, used a sorting paradigm and presented descriptions with related and unrelated co-occurrences under intentional category construction conditions. They found that $60 \%$ of the subjects sorted by related co-occurrences, whereas only $19 \%$ of the subjects sorted by unrelated cooccurrences. Indeed, at a general level, there is an abundance of evidence indicating that manipulations that increase the conceptual relatedness of features will make related materials easier to learn than unrelated materials in intentional learning conditions (e.g., Murphy \& Allopena, 1994; Pazzani, 1991; Wattenmaker et al., 1986). The influence of relatedness in intentional conditions was investigated further in the following experiment.

\section{EXPERIMENT 2B}

On the basis of the strength and consistency of previous research, it was assumed that prior knowledge would exert a clear influence with intentional encoding, and the primary question was whether this result would extend to incidental encoding. As was noted, however, no influence of relatedness was observed in the intentional conditions of Experiment 2A. The purpose of Experiment 2B was to attempt to replicate the advantage for conceptual re- latedness that was obtained in the intentional conditions of Experiment 1 and in previous experiments with intentional learning.

\section{Method}

Subjects. The subjects were 41 undergraduates, who participated in the experiment for course credit.

Stimuli and Procedure. The stimuli and procedure were identical to those used in the intentional conditions of Experiments 1 and 2, except that to provide a more stringent test of the relatedness effect in intentional conditions, only those randomizations with cooccurrences on nonadjacent dimensions were used (i.e., the D1-D4 and the D3-D5 randomizations but not the D2 -D3 or the D4-D5 randomizations). The results of Experiment 1 indicated that the subjects were less sensitive to co-occurrences on nonadjacent than on adjacent dimensions (e.g., the difference in the intentional conditions was .60 vs. .70). In addition, the advantage for having cooccurrences on adjacent dimensions appeared to be greater in the intentional-related than in the intentional-unrelated condition. Thus, using only those randomizations with nonadjacent co-occurrences should make it more difficult to encode the co-occurrences in either condition and might especially work against the intentional-related condition.

\section{Results and Discussion}

Fifty-two percent of the responses were consistent with the co-occurrences in the intentional-unrelated condition, whereas $64 \%$ of the responses were consistent with the co-occurrences in the intentional-related condition. This difference was significant $[t(39)=2.13, p<.05]$. In addition, significantly greater sensitivity to the co-occurrences was observed in the intentional-related condition than in the control-related condition $[t(68)=2.56, p<.025]$, but no significant difference was observed between the intentional-unrelated condition and the control-unrelated condition $[t(65)=0.47, p>.5]$. Thus, consistent with the results of Experiment 1 and with previous research, an influence of relatedness was observed with intentional encoding.

\section{GENERAL DISCUSSION}

Prior knowledge had a clear influence in incidental learning conditions. Greater sensitivity to conceptually related than to conceptually unrelated co-occurrences was observed with incidental learning, and conceptual relatedness increased sensitivity to co-occurrences at least as much in incidental as in intentional conditions. These results held for a variety of incidental tasks, including rating tasks, a recognition task, and an observation task. The results also generalized across descriptions of medical symptoms, animals, and people and occurred for cooccurrences at different levels of relatedness.

The results support the perspective that many types of knowledge-based influences will be strategy independent. Knowledge structures appeared to be spontaneously activated and applied during concept learning, and prior knowledge had the same type and degree of influence re- 
gardless of the nature of the encoding task. Thus, although influences of prior knowledge have previously only been observed with intentional concept-learning tasks, the results indicate that prior knowledge will have powerful influences on a variety of encoding tasks, including incidental learning.

The results indicated that people are especially sensitive to related co-occurrences, but sensitivity to unrelated co-occurrences was also observed. All six of the conditions with related co-occurrences and four of the six conditions with unrelated co-occurrences showed a clear tendency to preserve the co-occurrences in transfer decisions. People seem to be adept at capturing co-occurrence information in many different conditions (see, e.g., Alloy \& Tabachnik, 1984; Younger \& Cohen, 1986).

The extent to which incidental encoding will capture unrelated co-occurrences will probably depend on several factors, such as the nature of the incidental task, the distinctiveness of the exemplars, and the amount of exposure to the examples. Thus, although incidental encoding will not always capture unrelated co-occurrences (see, e.g., Anderson \& Fincham, 1996), if incidental encoding preserves information about individual exemplars, clear sensitivity to co-occurrence information is likely to occur (see, e.g., Wattenmaker, 1991, 1993; Wattenmaker et al., 1995).

\section{Generality of the Results}

The finding that background knowledge structures will be applied to concept learning regardless of the encoding task appears to have a fair amount of generality; as was noted, these results were observed with a range of different tasks and materials. There are a number of possible ways to extend these results, however. For example, there are many different types of knowledge, such as coherent theories, isolated facts, schemata, mental models, and abstract reasoning schemata, and the accessibility and application of some of these forms of knowledge might interact with encoding strategy. Likewise, in addition to relatively straightforward applications of prestored information, prior knowledge is useful for supporting complex inferences that can be used to connect features. It is possible that some encoding strategies will be more likely to lead to the formation of these inferences and elaborations than will other encoding strategies. In addition, in some cases, people might possess relevant knowledge, but this knowledge might be difficult to access. In cases in which people possess knowledge that is remotely related to the learning task or that is stored in a form that makes it difficult to access, differences in the application of the knowledge might occur as a function of encoding strategy. Some knowledge structures might be spontaneously retrieved and applied, whereas others might require strategy-mediated search and inferential processes to be applied. Additional research that investigates the influence of factors such as the nature and accessibility of relevant knowledge, the types of rules and relations that must be learned, and the nature of the encoding strategies that are used will help to elaborate the present results and reveal their generality as well as possible limitations.

Although future research will determine the generality of these results, intuitively it would seem that a lot of background knowledge about the world is easily accessible and will be spontaneously applied regardless of encoding strategy. For example, our typical mode of interacting with the environment is often not one of intentional, systematic problem solving, yet the wealth of knowledge that is needed to successfully comprehend, interpret, and interact with the environment is readily applied. Indeed, knowledge that supports understanding would often seem to be in the form of implicit theories (see, e.g., Dweck \& Leggett, 1988; Ross, 1989; Wattenmaker, 1995) or abstract schemata (see, e.g., Waldmann \& Holyoak, 1992; Waldmann et al., 1995), of which there is little if any awareness and no deliberate attempt to retrieve and apply during encoding. Thus, there seems to be a wealth of knowledge that is highly accessible and that will be spontaneously applied during concept learning.

The co-occurrences in these experiments could have been represented as explicit rules (see, e.g., Wattenmaker, 1993), represented as implicit rules (see, e.g., Reber, 1993), or indirectly preserved in memory representations (see, e.g., Perruchet, 1994; Vokey \& Brooks, 1992; Wattenmaker, 1993). Although any of these representations is possible, it would seem unlikely that the subjects in the incidental conditions formed explicit rules during learning. The co-occurrences were likely to have been salient at the end of learning, but given the tasks, it is unclear why explicit hypotheses or rules would have been developed in the incidental conditions.

\section{Summary}

The experiments in this paper merged two distinct lines of categorization research: research on knowledgebased influences and research on encoding strategies. Different encoding strategies often produce differences in classification in knowledge-poor contexts, and there were many reasons to expect knowledge-based influences to vary as a function of encoding strategy. Despite these potential differences, prior knowledge was found to have the same type and degree of influence with different encoding strategies. The results indicate that people will spontaneously apply background knowledge in a wide variety of encoding contexts and underscore the pervasiveness and power of the influence of background knowledge on concept formation.

\section{REFERENCES}

Alloy, L. B., \& TaBachniK, N. (1984). Assessment of covariations by humans and animals: The joint influence of prior expectations and current situational information. Psychological Review, 91, 112149.

Anderson, J. R., \& Fincham, J. M. (1996). Categorization and sensitivity to correlation. Journal of Experimental Psychology: Learning, Memory, \& Cognition, 22, 259-277. 
Billman, D., \& KnUtson, J. (1996). Unsupervised concept learning and value systematicity: A complex whole aids learning the parts. Journal of Experimental Psychology: Learning, Memory, \& Cognition, 22, 458-475.

BROOKS, L. (1978). Nonanalytic concept formation and memory for instances. In E. Rosch \& B. C. Lloyd (Eds.), Cognition and categorization (pp. 169-215). Hillsdale, NJ: Erlbaum.

BROOKS, L. (1987). Decentralized control of categorization: The role of prior processing episodes. In U. Neisser (Ed.), Concepts and conceptual development: Ecological and intellectual factors in categorization (pp. 141-174). Cambridge: Cambridge University Press.

DWECK, C. S., \& LEGGETT, E. L. (1988). A social-cognitive approach to motivation and personality. Psychological Review, 95, 256-273.

ELIo, R., \& ANDERSon, J. R. (1984). The effects of information order and learning mode on schema abstraction. Memory \& Cognition, 12, 20-30.

ELIO, R., \& LIN, K. (1994). Simulation models of the influence of learning mode and training variance on category learning. Cognitive Science, 18, 185-219.

ESTES, W. K. (1986). Memory storage and retrieval processes in category learning. Journal of Experimental Psychology: General, 115, 155-174.

GLUCK, M. A., \& BowER, G. H. (1988). Evaluating an adaptive network model of human learning. Journal of Memory \& Language, 27, 166-195.

HEIT, E. (1994). Models of the effects of prior knowledge on category learning. Journal of Experimental Psychology: Learning, Memory, \& Cognition, 20, 1264-1282.

Kemler Nelson, D. B. (1984). The effect of intention on what concepts are acquired. Journal of Verbal Learning \& Verbal Behavior, 23, 734-759.

Kemler Nelson, D. G. (1988). When category learning is holistic: A reply to Ward and Scott. Memory \& Cognition, 16, 79-84.

LEVINE, M. (1975). A cognitive theory of learning. Hillsdale, NJ: Erlbaum.

MALT, B. C., \& SMITH, E. E. (1984). Correlated properties in natural categories. Journal of Verbal Leaming \& Verbal Behavior, 23, 250-269.

Medin, D. L., Altom, M. W., Edelson, S. M., \& Freko, D. (1982). Correlated symptoms and simulated medical classification. Journal of Experimental Psychology: Human Learning \& Memory, 8, 37-50.

Medin, D. L., \& SMITH, E. E. (1981). Strategies and classification learning. Journal of Experimental Psychology: Human Learning \& Memory, 7, 241-253.

Medin, D. L., Wattenmaker, W. D., \& Hampson, S. E. (1987). Family resemblance, conceptual cohesiveness, and category construction. Cognitive Psychology, 19, 242-279.

MURPhY, G. L., \& ALLOPENA, P. D. (1994). The locus of knowledge effects in concept learning. Journal of Experimental Psychology. Learning, Memory, \& Cognition, 20, 904-919.

MuRPHY, G. L., \& MEDIN, D. L. (1985). The role of theories in conceptual coherence. Psychological Review, 92, 289-316.

MURPHY, G. L., \& WISNIEWSKI, E. J. (1989). Feature correlations in conceptual representations. In G. Tiberghian (Ed.), Advances in cognitive science: Vol. 2: Theory and applications (pp. 23-45). Chichester, U.K.: Ellis Horwood.

NosofSKY, R. M., CLARK, S. E., \& SHIN, H. J. (1989). Rules and exemplars in categorization, identification, and recognition. Journal of Experimental Psychology: Learning, Memory, \& Cognition, 15, 282-304.

Pazzani, M. J. (1991). Influence of prior knowledge on concept acquisition: Experimental and computational results. Journal of Experimental Psychology: Learning, Memory, \& Cognition, 17, 416-432.

Perruchet, P. (1994). Defining the knowledge units: Comment on Vokey \& Brooks-1992. Journal of Experimental Psychology: Learning, Memory, \& Cognition, 20, 223-238.

REBER, A. S. (1993). Implicit learning and tacit knowledge: An essay on the cognitive unconscious. Oxford: Oxford University Press.

Regehr, G., \& BRooks, L. R. (1993). Perceptual manifestations of an analytic structure: The priority of holistic individuation. Journal of Experimental Psychology: General, 122, 92-114.

Rosch, E. (1978). Principles of categorization. In E. Rosch \& B. B. Lloyd (Eds.), Cognition and categorization (pp. 27-48). Hillsdale, NJ: Erlbaum.
Ross, M. (1989). Relation of implicit theories to the construction of personal histories. Psychological Review, 96, 341-357.

SPALDING, T. L., \& MURPHY, G. L. (1996). Effects of background knowledge on category construction. Journal of Experimental Psychology: Learning, Memory, \& Cognition, 22, 528-538.

VOKEY, J. R., \& BROOKs, L. R. (1992). Salience of item knowledge in learning artificial grammars. Journal of Experimental Psychology: Learning, Memory, \& Cognition, 18, 328-344.

WaldmanN, M. R., \& Holyoak, K. J. (1992). Predictive and diagnostic learning within causal models: Asymmetries in cue competition Journal of Experimental Psychology: General, 121, 222-236.

Waldmann, M. R., Holyoak, K. J., \& Fratianne, A. (1995). Causa models and the acquisition of category structure. Journal of Experimental Psychology: General, 124, 181-206.

WARD, T. B. (1994). Structured imagination: The role of category structure in exemplar generation. Cognitive Psychology, 27, 1-40.

WARD, T. B., \& BECKER, A. H. (1992). Learning categories with and without trying: Does it make a difference? In B. Burns (Ed.), Percepts, concepts, and categories (pp. 451-491). Amsterdam: Elsevier.

WATTENMAKER, W. D. (1991). Learning modes, feature correlations, and memory-based categorization. Journal of Experimental Psychology: Learning, Memory, \& Cognition, 17, 908-923.

WATTENMAKER, W.D. (1992). Relational properties and memory-based category construction. Journal of Experimental Psychology: Learning, Memory, \& Cognition, 18, 1125-1138.

WATTENMAKER, W. D. (1993). Incidental concept learning, feature frequency, and correlated properties. Journal of Experimental Psychology: Learning, Memory, \& Cognition, 19, 203-222.

WATTENMAKER, W. D. (1995). Linear separability and knowledge structures: Integrating information in object and social categorization Cognitive Psychology, 28, 274-328.

Wattenmaker, W. D., Dewey, G. I., MurPhy, T. D., \& Medin, D. L. (1986). Linear separability and concept learning: Context, relational properties, and concept naturalness. Cognitive Psychology, 18, 158-194.

WatTenmaker, W. D., McQuaid, H. L., \& SchWerTz, S. J. (1995). Analogical versus rule-based classification. Memory \& Cognition, 23, 495-509.

Whit Lesea, B. W. A. (1987). Preservation of specific experiences in the representation of knowledge. Journal of Experimental Psychology: Learning, Memory, \& Cognition, 13, 3-17.

WISNIEWSKI, E. J., \& MEDIN, D. L. (1994). On the interaction of theory and data in concept learning. Cognitive Science, 18, 221-282.

YOUNGER, B. A., \& COHEN, L. B. (1986). Developmental changes in infants' perception of correlations among attributes. Child Development, 57, 803-815.

\section{APPENDIX A \\ Eight Learning Descriptions That Were Presented for One of the Randomizations in the Related Conditions of Experiments 1 and 2}

1. brittle bones; low sodium level; abdominal pain; hair loss; indigestion

2. reduced visual acuity; twisted ankle; abdominal pain; gum disease; indigestion

3. reduced visual acuity; twisted ankle; abdominal pain; hair loss; indigestion

4. brittle bones; low sodium level; abdominal pain; gum disease; indigestion

5. reduced visual acuity; low sodium level; fever; gum disease; fatigue

6. brittle bones; twisted ankle; fever; gum disease; fatigue

7. brittle bones; twisted ankle; fever; hair loss; fatigue

8. reduced visual acuity; low sodium level; fever; hair loss; fatigue

Note that the numbers in the left column are included to show the correspondence between the descriptions and the abstract notation in Table 1. 


\section{APPENDIX B}

Eight Learning Descriptions That Were

Presented for One of the Randomizations in the

Unrelated Conditions of Experiments 1 and 2

1. high red cell count; brittle bones; abdominal pain; twisted ankle; gum disease

2. low plasma level; reduced visual acuity; abdominal pain; low sodium level; gum disease

3. low plasma level; reduced visual acuity; abdominal pain; twisted ankle; gum disease

4. high red cell count; brittle bones; abdominal pain; low sodium level; gum disease

\section{APPENDIX B (Continued)}

5. low plasma level; brittle bones; fever; low sodium level; hair loss

6. high red cell count; reduced visual acuity; fever; low sodium level; hair loss

7. high red cell count; reduced visual acuity; fever; twisted ankle; hair loss

8. low plasma level; brittle bones; fever; twisted ankle; hair loss

Note that the numbers in the left column are included to show the correspondence between the descriptions and the abstract notation in Table 1 .

APPENDIX C

Results of the Individual Transfer Tests

in Experiments 1 and 2

\begin{tabular}{|c|c|c|c|c|c|c|c|c|c|c|c|c|}
\hline \multirow[b]{3}{*}{ Test } & \multicolumn{4}{|c|}{ Experiment 1} & \multicolumn{6}{|c|}{ Experiment 2} & \multirow{2}{*}{\multicolumn{2}{|c|}{ Control }} \\
\hline & \multicolumn{2}{|c|}{ Incid } & \multicolumn{2}{|c|}{ Inten } & \multicolumn{2}{|c|}{ Rec } & \multicolumn{2}{|c|}{ Obs } & \multicolumn{2}{|c|}{ Inten } & & \\
\hline & $\mathrm{U}$ & $\mathrm{R}$ & $\mathrm{U}$ & $\mathrm{R}$ & $\mathrm{U}$ & $\mathrm{R}$ & $\mathrm{U}$ & $\mathbf{R}$ & $\mathrm{U}$ & $\mathrm{R}$ & $\mathrm{U}$ & $\mathrm{R}$ \\
\hline 1 & .58 & .96 & .42 & .58 & .63 & .75 & .83 & .88 & .71 & .63 & .63 & .44 \\
\hline 2 & .67 & .75 & .54 & .83 & .63 & .83 & .54 & .71 & .63 & .58 & .54 & .60 \\
\hline 3 & .50 & .63 & .54 & .54 & .58 & .75 & .58 & .88 & .63 & .63 & .48 & .60 \\
\hline 4 & .54 & .92 & .63 & .79 & .54 & .79 & .54 & .79 & .67 & .79 & .38 & .54 \\
\hline 5 & .46 & .75 & .46 & .71 & .63 & .79 & .67 & .75 & .63 & .58 & .42 & .52 \\
\hline 6 & .67 & .83 & .50 & .71 & .46 & .92 & .54 & .79 & .83 & .67 & .56 & .50 \\
\hline 7 & .46 & .71 & .58 & .83 & .79 & .67 & .67 & .79 & .58 & .54 & .52 & .44 \\
\hline 8 & .50 & .96 & .67 & .71 & .67 & .75 & .58 & .71 & .63 & .75 & .60 & .52 \\
\hline 9 & .42 & .96 & .50 & .75 & .71 & .79 & .38 & .71 & .67 & .42 & .46 & .40 \\
\hline 10 & .67 & .75 & .50 & .79 & .67 & .83 & .50 & .71 & .75 & .50 & .31 & .48 \\
\hline 11 & .42 & .83 & .50 & .75 & .67 & .58 & .42 & .67 & .54 & .58 & .50 & .46 \\
\hline 12 & .63 & .83 & .54 & .75 & .67 & .83 & .63 & .63 & .67 & .67 & .50 & .54 \\
\hline 13 & .67 & .79 & .58 & .63 & .63 & .50 & .75 & .67 & .50 & .50 & .54 & .48 \\
\hline 14 & .58 & .88 & .46 & .79 & .79 & .71 & .67 & .63 & .63 & .71 & .58 & .48 \\
\hline 15 & .58 & .71 & .71 & .67 & .54 & .67 & .42 & .75 & .75 & .79 & .52 & .46 \\
\hline 16 & .58 & .75 & .67 & .83 & .67 & .83 & .42 & .50 & .50 & .50 & .54 & .44 \\
\hline 17 & .71 & .96 & .50 & .83 & .75 & .92 & .54 & .79 & .46 & .71 & .44 & .69 \\
\hline 18 & .58 & .83 & .54 & .79 & .71 & .83 & .71 & .79 & .71 & .71 & .40 & .67 \\
\hline 19 & .79 & .75 & .54 & .71 & .58 & .92 & .54 & .96 & .67 & .79 & .52 & .79 \\
\hline 20 & .58 & .88 & .63 & .88 & .67 & .92 & .71 & .88 & .75 & .75 & .52 & .63 \\
\hline
\end{tabular}

Note - Incid, incidental condition; Inten, intentional condition; Rec, recognition condition; Obs, observation condition; $U$, unrelated condition; $R$, related condition. Tests 1-16 represent fullinformation tests, and Tests 17-20 represent partial-information tests. 